\title{
Excited state quantum couplings and optical switching of an artificial molecule
}

\author{
K. Müller, ${ }^{1}$ G. Reithmaier, ${ }^{1}$ E. C. Clark, ${ }^{1}$ V. Jovanov, ${ }^{1}$ M. Bichler, ${ }^{1}$ H. J. Krenner, ${ }^{2}$ M. Betz, ${ }^{3}$ G. Abstreiter, ${ }^{1}$ and J. J. Finley ${ }^{1, *}$ \\ ${ }^{1}$ Walter Schottky Institut, Technische Universität München, Am Coulombwall 4, D-85748 Garching, Germany \\ ${ }^{2}$ Lehrstuhl für Experimentalphysik 1 and Augsburg Centre for Innovative Technologies (ACIT), Universität Augsburg, Universitätsstr. 1, \\ 86159 Augsburg, Germany \\ ${ }^{3}$ Experimentelle Physik 2, TU Dortmund, D-44221 Dortmund, Germany \\ (Received 26 April 2011; revised manuscript received 3 June 2011; published 11 August 2011)
}

\begin{abstract}
We optically probe the spectrum of ground and excited state transitions of an individual, electrically tunable self-assembled quantum dot molecule. Photocurrent absorption measurements show that the spatially direct neutral exciton transitions in the upper and lower dots are energetically separated by only $\sim 2 \mathrm{meV}$. Excited state transitions $\sim 8-16 \mathrm{meV}$ to higher energy exhibit pronounced anticrossings as the electric field is tuned due to the formation of hybridized electron states. We show that the observed excited state transitions occur between these hybridized electronic states and different hole states in the upper dot. By simultaneously pumping two different excited states with independent laser fields we demonstrate a strong ( $88 \%$ on-off contrast) laser-induced switching of the optical response. The results represent an electrically tunable, discrete coupled quantum system with a conditional optical response.
\end{abstract}

DOI: 10.1103/PhysRevB.84.081302

PACS number(s): 78.67.Hc, 81.07.Ta, 85.35.Be

Quantum dot (QD) nanostructures formed by strain driven self-assembly are ideal for solid-state quantum optics experiments due to their discrete optical spectrum, strong interaction with light and robust quantum coherence for both interband polarization ${ }^{1,2}$ and spin. ${ }^{3}$ Embedding them into electrically active devices allows for tuning of the transition frequency and control of charge occupancy. ${ }^{4}$ Vertically stacking produces more sophisticated nanostructures with coherent interdot coupling due to carrier tunneling. ${ }^{5-12}$ When combined with the potential to coherently manipulate excitons over ultrafast time scales using precisely timed laser and electrical control pulses, ${ }^{13-15}$ such systems raise exciting prospects for the operation of small-scale few qubit systems in a solid-state device. Very recently, conditional quantum dynamics for a single resonantly driven QD molecule (QDM) ${ }^{16}$ and spindependent quantum jumps have been observed. . $^{8}, 17$

In this Rapid Communication we employ photocurrent (PC) absorption, photoluminescence (PL) emission, and PL-excitation (PLE) spectroscopy to trace the spectrum of ground and excited state transitions of an individual selfassembled QD molecule as their character is electrically tuned from spatially direct to indirect. PC absorption allows us to identify the spatially direct neutral exciton transitions in both the upper $\left(X_{\mathrm{ud}}\right)$ and lower $\left(X_{\mathrm{ld}}\right)$ dots in the molecule. A number of excited state transitions are identified in PLE 8-16 meV above $X_{\mathrm{ud}}$. These excited states exhibit pronounced anticrossings (energy splitting $\Delta E \sim 3.2-3.5 \mathrm{meV}$ ) as the electric field $F$ is tuned. Excited state transitions are identified from voltage-dependent PLE measurements to correspond to transitions between these hybridized electronic states and different hole orbitals in the upper dot. By performing a multicolor experiment where the QDM is simultaneously excited with different frequency lasers, we demonstrate how the resonant excitation of indirect excitons or excitons in the lower quantum dot can be used to suppress the resonant excitation of the upper quantum dot, due to interdot Coulomb interactions. An on-off gating contrast up to $88 \%$ is observed, demonstrating a conditional optical response of an artificial molecule.

The sample consists of vertically stacked pairs of QDs separated by a 10-nm-thick GaAs spacer and embedded within the intrinsic region of a GaAs Schottky photodiode. ${ }^{9}$ Typical PL and PC measurements recorded at $T=4.2 \mathrm{~K}$ are presented in Fig. 1. For the PL measurement the sample was excited in the wetting layer at $1.49 \mathrm{eV}$. Typical electric-field-dependent PL from 18 to $32 \mathrm{kV} / \mathrm{cm}$ are presented in a grayscale contour plot representation in Fig. 1. The measurements show an anticrossing of two transitions arising from spatially direct and indirect excitons in the QDM where the hole is located in the upper dot. ${ }^{9}$ These optical transitions are depicted schematically in a single particle picture in the inset of Fig. 1: the direct exciton in the upper dot $X_{\mathrm{ud}}$ and the indirect exciton $X_{\text {ind }}$ with the hole in the upper dot and the electron in the lower dot. The indirect exciton exhibits a strong Stark shift due to the large static dipole. As the energies of the two states are tuned to resonance, electron-mediated tunnel coupling occurs that results in the formation of molecular bonding (lowerenergy) and antibonding (higher-energy) orbitals ${ }^{18}$ and the observed anticrossing. ${ }^{6}$ As $F$ increases beyond $\sim 25 \mathrm{kV} / \mathrm{cm}$, the intensity of the luminescence reduces as charge carriers escape from the QDM via tunneling and PC measurements can be performed with resonant optical excitation. Two prominent resonances are observed in PC, examples of which are presented in the left-hand panel of Fig. 1. The energy of these peaks are plotted in the main panel of Fig. 1 for $F=25-$ $31 \mathrm{kV} / \mathrm{cm}$. The transitions observed in PC arise from charge neutral excitons and show that the QD molecule exhibits two neutral exciton absorption resonances. As $F$ decreases, these two resonances clearly evolve into the two clear peaks observed in PL, labeled $X_{\mathrm{ld}}$ and $X_{\mathrm{ud}}$ on Fig. 1 . The state marked as $X_{\mathrm{ld}}$ is attributed to an exciton in the lower quantum dot of the molecule (depicted in blue in the inset of Fig. 1) while $X_{\text {ud }}$ is the direct exciton in the upper dot, assignments that are confirmed by the results presented below. 


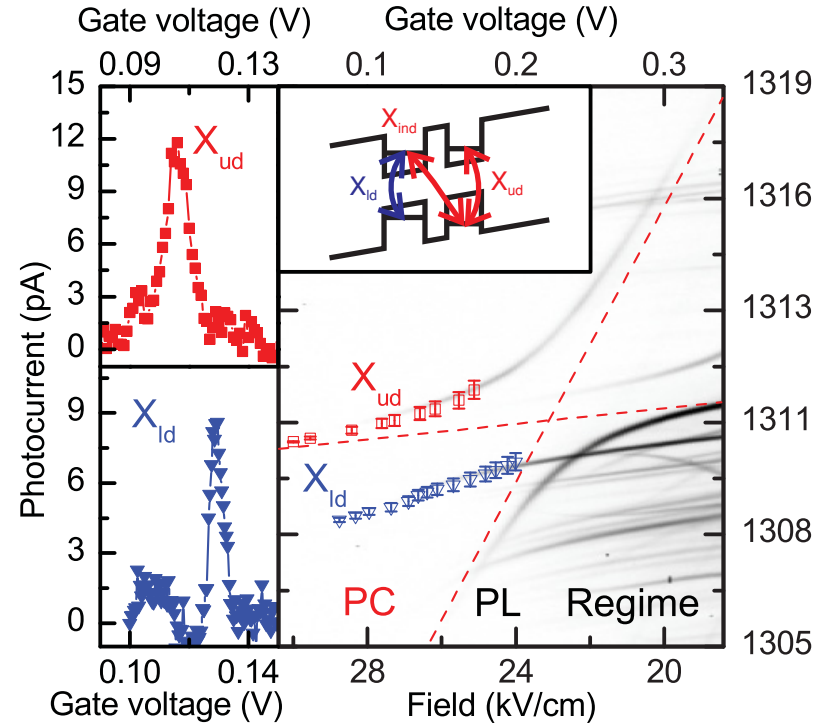

FIG. 1. (Color online) Combined result of an electric-fielddependent PL and PC measurement: The PL of the QDM as a function of the applied electric field is shown as a contour plot from 0 to $130 \mathrm{cps}$ from white to black on a logarithmic scale. The dashed red lines indicate the uncoupled neutral exciton in the upper dot and the indirect exciton as depicted schematically in the inset. The PC resonances are shown as red squares and blue triangles for the neutral exciton transitions in the upper and lower dots, respectively.

We conducted detailed PLE measurements to track the evolution of the excited state spectrum of the QDM as a function of $F$. A typical PLE scan detecting on $X_{\mathrm{ud}}$ is presented in Fig. 2(a) with $F$ fixed close to the anticrossing $(21.6 \mathrm{kV} / \mathrm{cm})$. Several discrete electronic resonances are observed in this region, the first four of which are labeled $e_{0 \mathrm{~B}}-h_{1}$, $e_{0 \mathrm{~B}}-h_{2}, e_{0 \mathrm{AB}}-h_{1}$, and $e_{0 \mathrm{AB}}-h_{2}$, in Fig. 2(a). This assignment anticipates the nature of these excited states corresponding to the electron being in the bonding (B) or antibonding (AB) molecular orbital while the hole occupies the first $\left(h_{1}\right)$ or second $\left(h_{2}\right)$ excited orbital state in the upper QD. These assignments are now justified by examining the electric-field dependence of the excited state resonances. Figure 2(b) shows the energy of the molecular ground states determined via PL and the first four excited states as a function of $F$ in the range $20-25 \mathrm{kV} / \mathrm{cm}$ measured using PLE. The first four excited states consist of two different pairs of lines color coded by the red and blue symbols in Fig. 2(b), each of which anticross at an electric field close to $22 \mathrm{kV} / \mathrm{cm}$. To analyze the observed excited state anticrossings in more detail and compare to the anticrossing of $X_{\mathrm{B}}$ and $X_{\mathrm{AB}}$ observed in PL, we plot the energy separation $(\Delta E)$ between the bonding and antibonding state of the ground states and the two excited state anticrossings in Fig. 2(c).

For all three anticrossings $\Delta E$ shows a similar hyperbolic behavior that can be fitted with

$$
\Delta E=\sqrt{\left(2 V_{e e}\right)^{2}+\left[e d\left(F-F_{0}\right)\right]^{2}},
$$

where $V_{e e}$ denotes the interdot tunnel coupling strength, $F_{0}$ is the field at which the states anticross, and $e d$ is the equivalent static dipole moment of the indirect exciton. ( $d$ is the distance between the centers of the electron and hole envelope functions.) Fits to the three anticrossings are (a)

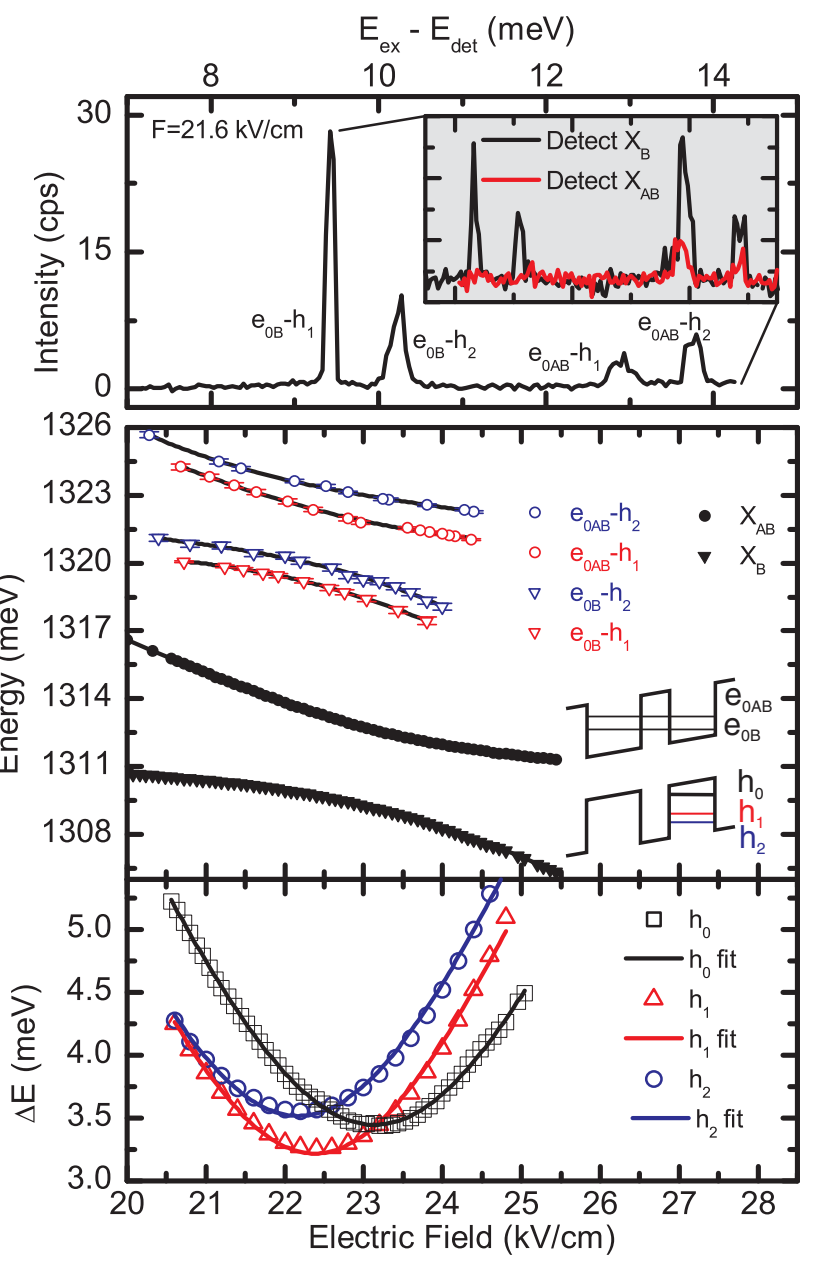

FIG. 2. (Color online) (a) Typical PLE scan detecting on $X_{\mathrm{B}}$. The inset compares PLE recorded at $F=23.2 \mathrm{kV} / \mathrm{cm}$ detecting either $X_{\mathrm{B}}$ (black) or $X_{\mathrm{AB}}$ (red), respectively. (b) Spectrum of QDM transitions determined by PL (black closed symbols) and PLE (black, red open symbols). States with bonding (antibonding) character are plotted as triangles (circles). (c) Energy separation $(\Delta E)$ between corresponding anticrossing states, fits using Eq. (1).

presented as lines in Fig. 2(c) and the extracted values of $F_{0}$ and $d$ are summarized in Table I. For each anticrossing the extracted values of $d$ vary only slightly and are fully consistent with the dot height of $5 \mathrm{~nm}$ and the separation of $10 \mathrm{~nm}$. Both $V_{e e}$ and $F_{0}$ remain practically unchanged for the transitions involving $h_{0}, h_{1}$, and $h_{2}$, providing evidence that they arise from transitions between the same electron-mediated anticrossing and different hole levels. This expectation is confirmed by PLE measurements performed close to $F_{0}$, detecting on either $X_{\mathrm{AB}}$ or $X_{\mathrm{B}}$, respectively. Typical results are presented in Fig. 2(a) (inset). When detecting on $X_{\mathrm{AB}}$ (red curve), transitions are only observed for $e_{0 \mathrm{AB}}-h_{1}$ and $e_{0 \mathrm{AB}}-h_{2}$, while $e_{0 \mathrm{~B}}-h_{1}$ and $e_{0 \mathrm{~B}}-h_{2}$ are absent. This arises since the electron populates the lower-energy bonding level and thermal activation into the higher-energy bonding level is unlikely since $2 V_{e e} \gg k_{B} T{ }^{19}$ In contrast, upon exciting states with bonding electron character, all four resonances are observed due to phonon-mediated thermalization from antibonding to bonding electron states. ${ }^{19}$ The small differences between $F_{0}$ arise from the Coulomb interactions between the 
TABLE I. Results of the fits of $\Delta E$ from Fig. 2(c) with Eq. (1).

\begin{tabular}{lccc}
\hline \hline Hole state & $2 V_{e e}(\mathrm{meV})$ & $F_{0}(\mathrm{kV} / \mathrm{cm})$ & $d(\mathrm{~nm})$ \\
\hline$h_{0}$ & $3.4 \pm 0.1$ & $23.1 \pm 0.1$ & $15.3 \pm 0.1$ \\
$h_{1}$ & $3.2 \pm 0.1$ & $22.4 \pm 0.1$ & $15.8 \pm 0.2$ \\
$h_{2}$ & $3.5 \pm 0.1$ & $22.2 \pm 0.1$ & $15.9 \pm 0.2$ \\
\hline \hline
\end{tabular}

various hole orbital states. Compared to the ground state with $F_{0}=23.1 \pm 0.1 \mathrm{kV} / \mathrm{cm}$, the critical field of the excited state transitions are shifted by $-0.7 \pm 0.2$ and $-0.9 \pm 0.2 \mathrm{kV} / \mathrm{cm}$. This can be converted to an energy difference of $1.1 \pm 0.3$ and $1.4 \pm 0.4 \mathrm{meV}$ using $d=15.8 \pm 0.2$ and $15.9 \pm 0.2 \mathrm{~nm}$, respectively. These values correspond to less than $10 \%$ of the total attractive $e-h$ Coulomb interaction that has been estimated to be $\sim 22 \mathrm{meV}$ for similar samples. ${ }^{9}$ Thus, the Coulomb shifts between the different valence-band states represent a small perturbation and we conclude that these observations provide strong evidence that the four excited states shown in Fig. 2(b) take place between an electron in either the lowest-energy bonding or antibonding levels and different hole states in the upper dot, as depicted in the inset of Fig. 2(b). The energy splitting between the lowest-energy hole state and the first two excited states is 9.5 and $10.5 \mathrm{meV}$, respectively.

Away from resonance for $F<F_{0}$ the bonding ground state as well as the first two excited states $e_{0 \mathrm{~B}}-h_{1}$ and $e_{0 \mathrm{~B}}-h_{2}$ have predominant direct character, while the third and fourth

(a)
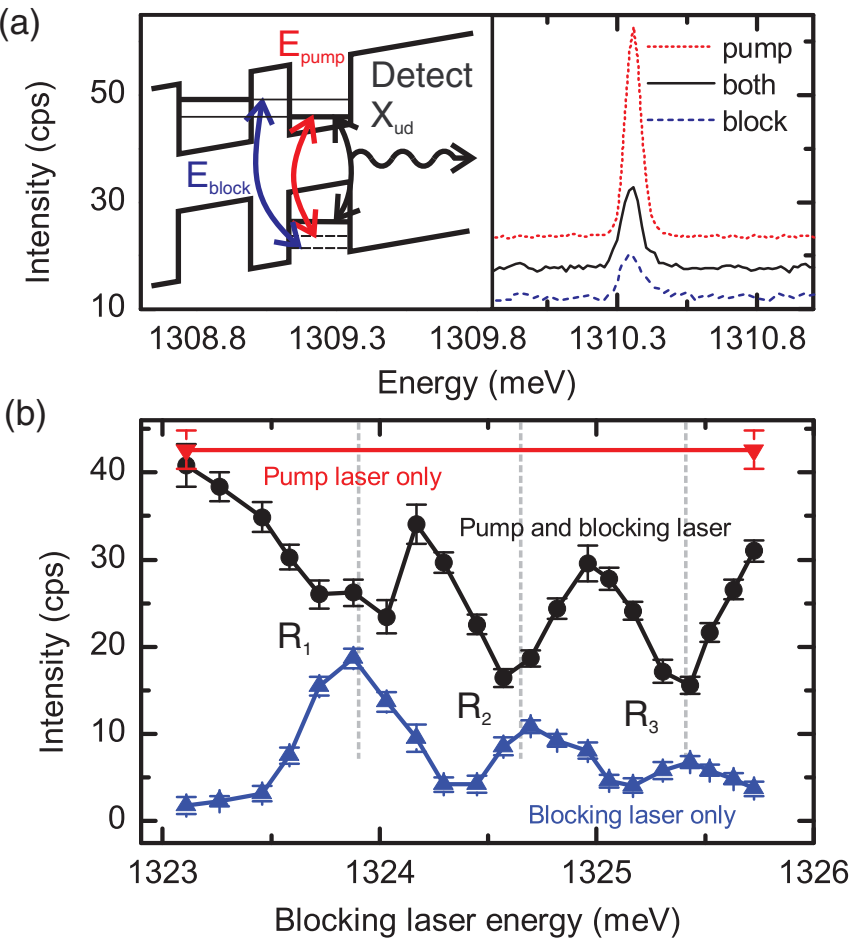

FIG. 3. (Color online) (a) PL intensity of $X_{\text {ud }}$ for the excitation of a resonance with direct character (pump, red), indirect character (block, blue), and both lasers (black). PL from the excitation of the direct resonance is quenched due to the presence of the block laser. (b) Intensity of $X_{\text {ud }}$ as a function of the block laser energy. Whenever the block laser hits an excited state with indirect character, the PL from the upper dot is decreased. excited states $e_{0 \mathrm{AB}}-h_{1}$ and $e_{0 \mathrm{AB}}-h_{2}$ have indirect character. Therefore, exciting $e_{0 \mathrm{~B}}-h_{1}$ and $e_{0 \mathrm{~B}}-h_{2}$ is expected to populate $X_{\mathrm{ud}}$ while exciting $e_{0 \mathrm{AB}}-h_{1}$ and $e_{0 \mathrm{AB}}-h_{2}$ generates an indirect exciton $X_{\text {ind }}$. This is used to test whether the QDM exhibits a conditional optical response with a scheme illustrated in the inset of Fig. 3(a). With the condition $F<F_{0}$ as described above the system is resonantly excited by either one laser or two lasers simultaneously. In the two laser experiment, the first laser termed pump is resonant with $e_{0 \mathrm{~B}}-h_{1}$, as indicated by the red arrow on the inset of Fig. 3(a) (middle arrow). As discussed above, an exciton created by laser absorption will primarily relax to the $X_{\mathrm{ud}}$ ground state before PL is measured via phonon-mediated processes. ${ }^{20} \mathrm{~A}$ second laser, termed block, is tuned into resonance with excited states that have a predominantly indirect character in order to generate excitons with indirect character $X_{\text {ind }}$. If the absorption of the blocking laser is more efficient than that of the pump, then the QDM will be driven into an indirect exciton state and the absorption of the pump laser is suppressed since absorption

(a)

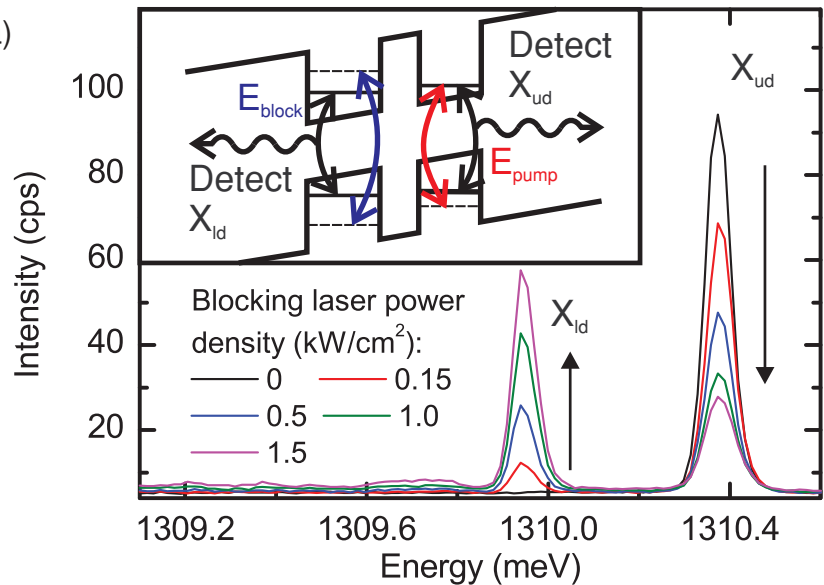

(b)

(c)

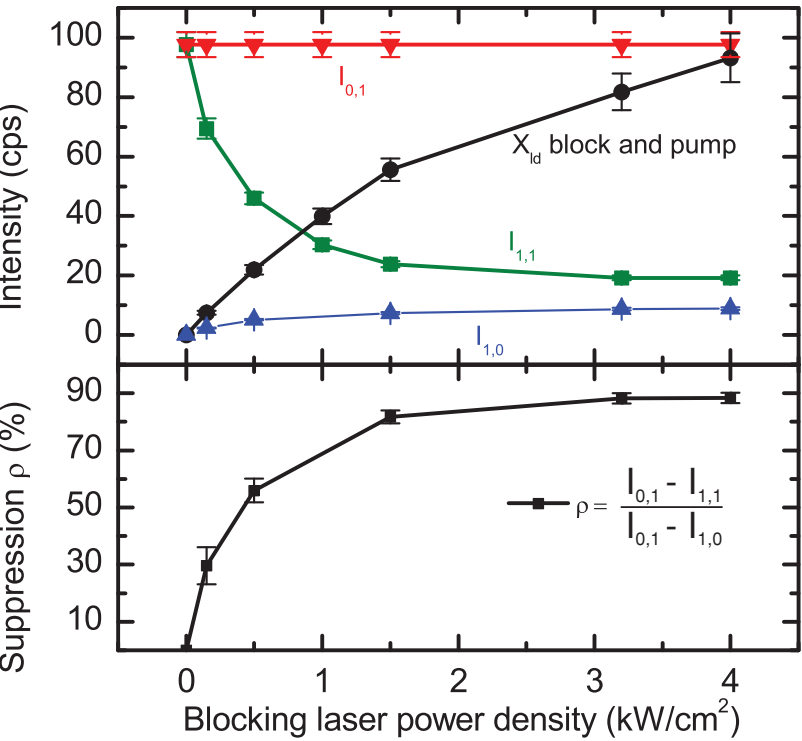

FIG. 4. (Color online) PL of the upper and lower quantum dot as a function of the blocking laser power. Resonant excitation of an exciton in the upper dot is blocked by the presence of an exciton in the lower dot due to the Coulomb interaction. (b) Intensity of the PL of the upper and lower dot and (c) suppression ratio $\rho$ as a function of the blocking laser power. 
shifts to a biexcitonic state of the system. Typical results of such a measurements are presented in Fig. 3(a), which shows the PL spectrum recorded from $X_{\mathrm{ud}}$ for $F=21.05 \mathrm{kV} / \mathrm{cm}$ when the pump laser only $\left(0.5 \mathrm{~kW} / \mathrm{cm}^{2}\right)$ is applied (red curve). This is compared with the situation when the system is excited only by the blocking laser $\left(5 \mathrm{~kW} / \mathrm{cm}^{2}\right.$, blue curve $)$ and when both lasers are applied simultaneously (black curve).

The intensity of $X_{\mathrm{ud}}$ clearly reduces strongly when both lasers are applied simultaneously. We scanned the energy of the blocking laser over the spectrum of excited states from 1322 to $1326 \mathrm{meV}$. The result of this experiment is shown in Fig. 3(b) comparing the intensity of $X_{\mathrm{ud}}$ as a function of the blocking laser energy for the pump laser only (red curve), the blocking laser only (blue curve), and with both blocking and pump lasers applied simultaneously (black curve). Three resonances, labeled $R_{1}, R_{2}$, and $R_{3}$ in Fig. 3(b) can be clearly seen. At these resonances PL is observed from $X_{\mathrm{ud}}$ following excitation with the blocking laser only. The PL signal for excitation with both lasers shows a series of dips for an excitation with the pump laser only. $R_{1}$ and $R_{2}$ coincide precisely with the PLE resonances $e_{0 \mathrm{AB}}-h_{1}$ and $e_{0 \mathrm{AB}}-h_{2}$, presented in Fig. 2(b), demonstrating that the blocking laser can be used to suppress the absorption of the pump beam.

We now turn to an experimental scheme where an excitation in one of the dots forming the molecule was used to block absorption in the other. The scheme for this experiment is illustrated schematically in the inset of Fig. 4(a). An exciton with predominantly direct character can be excited in the upper dot via its first excited hole state (red arrow). If the blocking laser is tuned to a direct exciton transition in the lower dot (blue arrow) while the pump field is simultaneously on the energy of the upper dot absorption shifts to the spatially separated biexciton. Thus, the absorption of the pump laser is switched off and thus PL from $X_{\text {ud }}$ can be optically gated on and off. The result of such a measurement is presented in Fig. 4(a), which shows the PL intensity of $X_{\text {ud }}$ and $X_{\mathrm{ld}}$ for $F=20.8 \mathrm{kV} / \mathrm{cm}$, a pump laser power density of $1 \mathrm{~kW} / \mathrm{cm}^{2}$, and different blocking laser power densities from 0 to $1.5 \mathrm{~kW} / \mathrm{cm}^{2}$.
In the absence of the blocking laser, only PL from $X_{\mathrm{ud}}$ can be seen with an intensity of $98 \pm 4$ cps. However, upon increasing the power of the blocking laser, we observe a pronounced decrease in the intensity of $X_{\mathrm{ud}}$, while emission from $X_{\mathrm{ld}}$ emerges and gradually increases in intensity. These observations clearly demonstrate a conditional optical response, blocking of $X_{\text {ud }}$ induced by direct excitation of $X_{\mathrm{ld}}$. To quantitatively analyze the effect of the blocking laser on the PL signal from the QDM, we define the intensity of $X_{\text {ud }}$ subject to the combined pump and blocking lasers as $I_{\text {block,pump }}$ : Here $I_{1,1}$ corresponds to the intensity measured when blocking and pump lasers are switched on, $I_{0,1}$ is the intensity with only the pump laser, etc. These three intensities are plotted together with the intensity of the lower dot in Fig. 4(b) as a function of the blocking laser power. We measure $I_{0,1}=98 \pm 4 \mathrm{cps}$ (red triangles) and $I_{1,1}=19 \pm 1 \mathrm{cps}$ (green squares) as the intensity of the blocking laser is increased to $4 \mathrm{~kW} / \mathrm{cm}$. When only the blocking laser is applied, we measure $I_{1,0}=9 \pm 1 \mathrm{cps}$ (blue triangles), from which we obtain the suppression $\rho=\frac{I_{0,1}-I_{1,1}}{I_{0,1}-I_{1,0}} \sim 88 \pm 2 \%$. The dependence of $\rho$ on the blocking laser power is presented in Fig. 4(c). The reason why the blocking laser-induced $\mathrm{PL}$ from $X_{\mathrm{ud}}$ results from the fact that the resonance used for efficiently exciting $X_{\mathrm{ld}}$ is $22.3 \mathrm{meV}$ higher than the ground state of $X_{\mathrm{ld}}$. Therefore, relaxation from this excited state to the ground state of $X_{\mathrm{ud}}$ is possible due to tunneling of both charge carriers.

In summary, we probed the spectrum of ground and excited state transitions in an individual, electrically tunable artificial molecule. Excited state transitions were identified between hybridized electron states having bonding or antibonding character and different excited hole states. By simultaneously pumping different discrete optical transitions, we demonstrated a conditional optical response with an on-off gating fidelity of $88 \pm 2 \%$.

We gratefully acknowledge financial support of the DFG via SFB-631, Nanosystems Initiative Munich and the Emmy Noether Program (H.J.K.) and the EU via SOLID.

\footnotetext{
*finley@wsi.tum.de

${ }^{1}$ P. Borri, W. Langbein, S. Schneider, U. Woggon, R. L. Sellin, D. Ouyang, and D. Bimberg, Phys. Rev. Lett. 87, 0157401 (2001).

${ }^{2}$ P. Borri, W. Langbein, U. Woggon, M. Schwab, M. Bayer, S. Fafard, Z. Wasilewski, and P. Hawrylak, Phys. Rev. Lett. 91, 0267401 (2003).

${ }^{3}$ A. Greilich et al., Nat. Phys. 5, 262 (2009).

${ }^{4}$ R. J. Warburton et al., Nature (London) 405, 926 (2000).

${ }^{5}$ E. Stinaff et al., Science 311, 636 (2006).

${ }^{6}$ H. J. Krenner, E. C. Clark, T. Nakaoka, M. Bichler, C. Scheurer, G. Abstreiter, and J. J. Finley, Phys. Rev. Lett. 97, 076403 (2006).

${ }^{7}$ M. F. Doty, M. Scheibner, A. S. Bracker, I. V. Ponomarev, T. L. Reinecke, and D. Gammon, Phys. Rev. B 78, 115316 (2008).

${ }^{8}$ D. Kim et al., Nat. Phys. 7, 223 (2011)

${ }^{9}$ H. J. Krenner, M. Sabathil, E. C. Clark, A. Kress, D. Schuh, M. Bichler, G. Abstreiter, and J. J. Finley, Phys. Rev. Lett. 94, 057402 (2005).

${ }^{10}$ A. S. Bracker et al., Appl. Phys. Lett. 89, 233110 (2006).
}

${ }^{11}$ M. Scheibner, I. V. Ponomarev, E. A. Stinaff, M. F. Doty, A. S. Bracker, C. S. Hellberg, T. L. Reinecke, and D. Gammon, Phys. Rev. Lett. 99, 197402 (2007).

${ }^{12}$ M. Scheibner et al., Nat. Phys. 4, 291 (2008).

${ }^{13}$ A. Zrenner et al., Nature (London) 418, 612 (2002).

${ }^{14}$ M. Zecherle, C. Ruppert, E. C. Clark, G. Abstreiter, J. J. Finley, and M. Betz, Phys. Rev. B 82, 125314 (2010).

${ }^{15}$ S. M. de Vasconcellos et al., Nat. Photon. 4, (2010).

${ }^{16}$ L. Robledo et al., Science 320, 772 (2008).

${ }^{17}$ A. N. Vamivakas et al., Nature (London) 467, 297 (2010).

${ }^{18}$ Assignment motivated by the comparison with natural diatomic molecular states.

${ }^{19}$ T. Nakaoka, E. C. Clark, H. J. Krenner, M. Sabathil, M. Bichler, Y. Arakawa, G. Abstreiter, and J. J. Finley, Phys. Rev. B 74, 121305 (2006).

${ }^{20}$ B. Ohnesorge, M. Albrecht, J. Oshinowo, A. Forchel, and Y. Arakawa, Phys. Rev. B 54, 11532 (1996). 